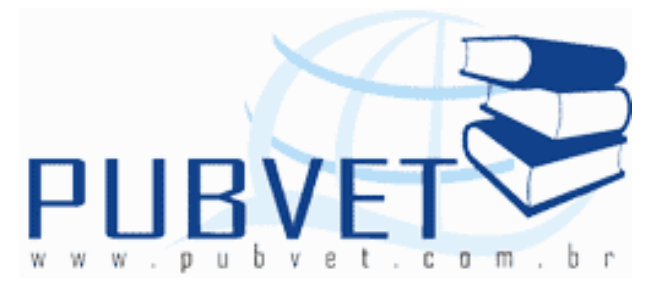

PUBVET, Publicações em Medicina Veterinária e Zootecnia.

\title{
Comprimentos parciais e totais de intestino de gatos domésticos Felis catus domesticus - (Linnaeus, 1758).
}

\footnotetext{
André Luiz Quagliatto Santos ${ }^{1}$, Lorena Tannús Menezes ${ }^{2}$, Arthur Paulino Sanzo Kaminishi ${ }^{2}$, Tatiana Grillo Leonardo ${ }^{2}$, Liliane Rangel Nascimento ${ }^{2}$, Tablita Silva Malta $^{3}$, Antônio Maximiano Neto ${ }^{3}$
}

Laboratório de Ensino e Pesquisas em Animais Silvestres - LAPAS, FAMEV/UFU, e-mail: quagliatto@famev.ufu.br 1. Docente. 2. Mestrandos. 3. Graduandos.

\section{Resumo}

Até o presente momento, tem sido estudado o comprimento de partes do intestino bem como o comprimento intestinal total de varias espécies de mamíferos domésticos e silvestres. Neste estudo foram ultilizados 16 gatos domésticos (Felis catus domesticus), sendo 8 machos e 8 fêmeas, provenientes da cidade de Uberaba, Minas Gerais, Brasil. Após a remoção dos órgãos dos animais o intestino foi isolado e as partes foram mensuradas. Os valores encontrados para comprimento médio, máximo e mínimo foram respectivamente: duodeno $-9,20 \mathrm{~cm}(13,50 \mathrm{~cm}$ e 5,80 cm); jejuno - 109,56 $\mathrm{cm}(132,60 \mathrm{~cm}$ e $72,70 \mathrm{~cm})$; íleo $-2,49 \mathrm{~cm}(4,00 \mathrm{~cm}$ e $1,50 \mathrm{~cm})$; ceco $-1,68$ $\mathrm{cm}(2,50 \mathrm{~cm}$ e $1,00 \mathrm{~cm})$; cólon ascendente $-4,49 \mathrm{~cm}(8,00 \mathrm{~cm}$ e 2,10 cm); cólon transverso $-1,82 \mathrm{~cm}(4,10 \mathrm{~cm}$ e $0,60 \mathrm{~cm})$; cólon descendente $-9,22 \mathrm{~cm}$ $(13,40 \mathrm{~cm}$ e $7,20 \mathrm{~cm})$; reto $-3,46 \mathrm{~cm}(4,50 \mathrm{~cm}$ e $3,00 \mathrm{~cm})$ e comprimento 
SANTOS, A.L.Q. et al. Comprimentos parciais e totais de intestino de gatos domésticos Felis catus domesticus - (Linnaeus, 1758). PUBVET, Londrina, V. 5, N. 14, Ed. 161, Art. 1092, 2011.

total - 147,20 cm $(237,50 \mathrm{~cm}$ e $100,20 \mathrm{~cm})$. Os machos apresentam o comprimento intestinal total maior que das fêmeas (164,90 e 129,57 respectivamente).

Palavras-chave: Felis catus domesticus, anatomia, biometria, intestino

\title{
Partial and total intestinal length of domestic cats Felis catus domesticus - (Linnaeus, 1758).
}

\begin{abstract}
Until this present moment has been studied the length of intestinal segments and the total intestinal length of several species of wild and domestic mammals. In this study used 16 domestic cat (Felis catus domesticus), being 8 males and 8 females, from the city of Uberaba, Minas Gerais, Brazil. After remove the organs of the animals, the intestine was isolated and the parts were measured. The following values was found for medium, maximum and minimum lengths, respectively: duodenum $-9.20 \mathrm{~cm}(13.50 \mathrm{~cm}$ and $5.80 \mathrm{~cm})$; jejunum - $109.56 \mathrm{~cm}(132.60 \mathrm{~cm}$ and $72.70 \mathrm{~cm})$; ileum $-2.49 \mathrm{~cm}(4.00 \mathrm{~cm}$ and $1.50 \mathrm{~cm})$; cecum $-1.68 \mathrm{~cm}(2.50 \mathrm{~cm}$ and $1.00 \mathrm{~cm})$; ascending colon $4.49 \mathrm{~cm}(8.00 \mathrm{~cm}$ and $2.10 \mathrm{~cm})$; transverse colon $-1.82 \mathrm{~cm}(4.10 \mathrm{~cm}$ and $0.60 \mathrm{~cm})$; descending colon $-9.22 \mathrm{~cm}(13.40 \mathrm{~cm}$ and $-7.20 \mathrm{~cm})$; rectum $3.46 \mathrm{~cm}(4.50 \mathrm{~cm}$ and $3.00 \mathrm{~cm})$ and the total lenght $-147.20 \mathrm{~cm}(237.50 \mathrm{~cm}$ and $100.20 \mathrm{~cm}$ ). Males have a longer length of intestine than females (164.90 $\mathrm{cm}$ and $129.57 \mathrm{~cm}$ respectively).
\end{abstract}

Keywords: Felis catus domesticus, anatomy, biometry, intestine.

\section{INTRODUÇÃO}

As dimensões dos intestinos são de considerável interesse para investigadores em diversos campos, parasitologistas, por exemplo, freqüentemente necessitam de uma acurada mensuração do comprimento intestinal para determinar a distribuição linear de helmintos ao longo do tubo digestivo (Mettrick e Podesta, 1974; Crompton e Nesheim, 1976). Em estudos 
SANTOS, A.L.Q. et al. Comprimentos parciais e totais de intestino de gatos domésticos Felis catus domesticus - (Linnaeus, 1758). PUBVET, Londrina, V. 5, N. 14, Ed. 161, Art. 1092, 2011.

de nutrição animal e fisiologia intestinal também levam em consideração as mensurações intestinais como índices para alterações induzidas pela dieta na função e morfologia intestinal.

As informações sobre aspectos morfológicos e biométricos intestinais fornecem subsídios para pesquisas com enfoque fisiológico e nutricional, visando uma melhoria na dieta alimentar dos animais (González-Jiménez, 1977).

Segundo Reece (1996) determinadas partes do tubo digestivo do animal são mais desenvolvidas, caracterizando uma adaptação evolutiva em relação a sua dieta. Enquanto o cão, animal carnívoro, tem ceco insignificante; o cavalo, animal herbívoro, tem ceco volumoso. O porco, que é onívoro, não somente possui um intestino delgado relativamente longo para digestão e absorção dos alimentos que não necessitem de fermentação, como também, cólon ascendente desenvolvido, transformado em espiral, em que a fermentação da porção fibrosa da dieta é realizada.

Vários autores têm estudado a longitude intestinal de diferentes mamíferos domésticos e silvestres, Paiva e Borelli (1977) avaliaram longitude total do intestino de bovinos azebuado; Paiva, Borelli e Pedutti Neto (1977) estudaram comprimento total do intestino de bovinos de origem europeia; Pereira et al (1986) avaliaram o intestino de suínos; Pereira et al (1988) estudaram o intestino do ratão do banhado; Santos et al (1998) e Amorim et al (2002) avaliaram o comprimento total do intestino de coelhos e Rodrigues et al (2006) avaliou o intestino delgado de capivara.

Foram encontrados poucos trabalhos com a biometria intestinal em gatos, Dyce et al (1990) citam genericamente que o intestino delgado é formado pelo duodeno, jejuno e íleo, onde citam também apenas o comprimento da primeira porção que é de $25 \mathrm{~cm}$ em média.Segundo Getty (1986) o intestino delgado de carnívoros é dividido em duodeno, jejuno e íleo e possui um comprimento médio de cerca de $400 \mathrm{~cm}$, o intestino grosso por sua vez têm em média 60 a $75 \mathrm{~cm}$. 
SANTOS, A.L.Q. et al. Comprimentos parciais e totais de intestino de gatos domésticos Felis catus domesticus - (Linnaeus, 1758). PUBVET, Londrina, V. 5, N. 14, Ed. 161, Art. 1092, 2011.

Considerando a importância dos gatos como animais de estimação e muitas vezes sendo considerado um membro efetivo da família, a necessidade de conhecimentos anatômicos e biométricos para possíveis intervenções cirúrgicas no abdômen destes animais, principalmente em laparotomias exploratórias justificam o presente trabalho.

O objetivo do trabalho é o estudo dos comprimentos parciais e totais do intestino do gato doméstico (Felis catus domesticus) bem como avaliar possíveis diferenças entre o comprimento intestinal de machos e fêmeas.

\section{MATERIAL E MÉTODOS}

Foram utilizados 16 gatos, sendo 8 machos e 8 fêmeas provenientes do Centro de Controle de Zoonoses de Uberaba-MG. Procedeu-se a abertura da cavidade abdominal, na linha alba, com instrumentos cirúrgicos convencionais. Após a identificação dos órgãos internos promoveu-se a retirada e o isolamento do intestino, em seguida, com o intestino sobre uma superfície horizontal, realizou-se as mensurações dos diversos segmentos intestinais resultando em suas medidas parciais e totais.

\section{RESULTADOS E DISCUSSÃO}

No trabalho de Santos et al (1998), que estuda o comprimento intestinal de coelhos (Oryctolagus cuniculus) foi encontrado os valores médio, máximo e mínimo para o duodeno de $52,70 \mathrm{~cm}(68,00 \mathrm{~cm}$ e $34,00 \mathrm{~cm})$, enquanto que nos gatos encontrou-se $9,20 \mathrm{~cm}(13,50 \mathrm{~cm}$ e $5,80 \mathrm{~cm})$, respectivamente (Tabela 1). E segundo Dyce et al (1990) o duodeno apresenta $25 \mathrm{~cm}$.

Santos et al (1998) mediram o conjunto jejuno e íleo encontrando $216,50 \mathrm{~cm}(254,00 \mathrm{~cm}$ e $182,00 \mathrm{~cm})$, foi obtido nos gatos: para o jejuno $109,56 \mathrm{~cm}(132,60 \mathrm{~cm}$ e $72,70 \mathrm{~cm})$ e para o íleo $2,49 \mathrm{~cm}(4,00 \mathrm{~cm}$ e $1,50 \mathrm{~cm}$ (Tabela 1) (Figura 1). Segundo Getty (1986) os carnívoros apresentam em média $400 \mathrm{~cm}$ de duodeno, jejuno e íleo.

Ainda em Santos et al (1998) encontraram para o ceco de coelhos 44,40 $\mathrm{cm}(50,00 \mathrm{~cm}$ e $38,00 \mathrm{~cm})$, enquanto em gatos foi encontrado $1,68 \mathrm{~cm}(2,50$ 
$\mathrm{cm}$ e 1,00 cm) (Tabela 1). Santos et al (1998), obtiveram para o conjunto cólon e reto as seguintes medidas: $107,80 \mathrm{~cm}(130,00 \mathrm{~cm}$ e $77,00 \mathrm{~cm})$, neste trabalho foi encontrado para o cólon ascendente $4,49 \mathrm{~cm}(8,00 \mathrm{~cm}$ e $2,10 \mathrm{~cm})$, para o cólon transverso $1,82 \mathrm{~cm}(4,10 \mathrm{~cm}$ e 0,60 cm), para o cólon descendente $9,22 \mathrm{~cm}(13,40 \mathrm{~cm}$ e $7,20 \mathrm{~cm})$ e para o reto $3,46 \mathrm{~cm}(4,50 \mathrm{~cm}$ e 3,00) (Tabela 1) (Figura 2). Getty (1986) aponta que o intestino grosso de carnívoros tem o comprimento de $60-75 \mathrm{~cm}$.

Quanto ao comprimento total do intestino em coelhos, Santos et al

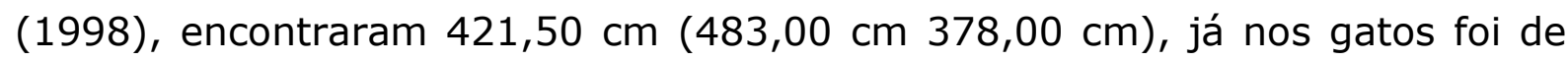
$147,24 \mathrm{~cm}(237,50 \mathrm{~cm}$ e $100,20 \mathrm{~cm})$ (Tabela1).

Os machos apresentaram comprimento maior em todos os segmentos intestinais totalizando $164,9 \mathrm{~cm}$ de intestino, enquanto que nas fêmeas foi encontrado um comprimento total médio de 129,57 cm (Tabela 2).

Tabela 1- Comprimento médio em centímetros dos diversos segmentos intestinais e comprimento total médio do intestino de gatos (n-16).

\begin{tabular}{lccc}
\hline & $\begin{array}{c}\text { Valores } \\
\text { médios }\end{array}$ & $\begin{array}{c}\text { Valores } \\
\text { máximos }\end{array}$ & $\begin{array}{c}\text { Valores } \\
\text { mínimos }\end{array}$ \\
\hline Duodeno & 9,20 & 13,50 & 5,80 \\
Jejuno & 109,56 & 132,60 & 72,70 \\
Íleo & 2,49 & 4,00 & 1,50 \\
Ceco & 1,68 & 2,50 & 1,00 \\
Cólon & 4,49 & 8,00 & 2,10 \\
ascendente & & & \\
Cólon transverso & 1,82 & 4,10 & 0,60 \\
Cólon & 9,22 & 13,40 & 7,20 \\
descendente & & & 3,00 \\
Reto & 3,40 & 4,50 & $\mathbf{1 0 0 , 2 0}$ \\
Comprimento & $\mathbf{1 4 7 , 2 4}$ & $\mathbf{2 3 7 , 5 0}$ & \\
total & & & \\
\hline
\end{tabular}


SANTOS, A.L.Q. et al. Comprimentos parciais e totais de intestino de gatos domésticos Felis catus domesticus - (Linnaeus, 1758). PUBVET, Londrina, V. 5, N. 14, Ed. 161, Art. 1092, 2011.

Segundo Reece (1996) cada animal apresenta determinado desenvolvimento do tubo digestivo em função da adaptação evolutiva à sua dieta, considerando que coelhos são herbívoros e gatos carnívoros, era de se esperar que apesar de terem dimensões corporais semelhantes apresentem biometria intestinal diferentes.

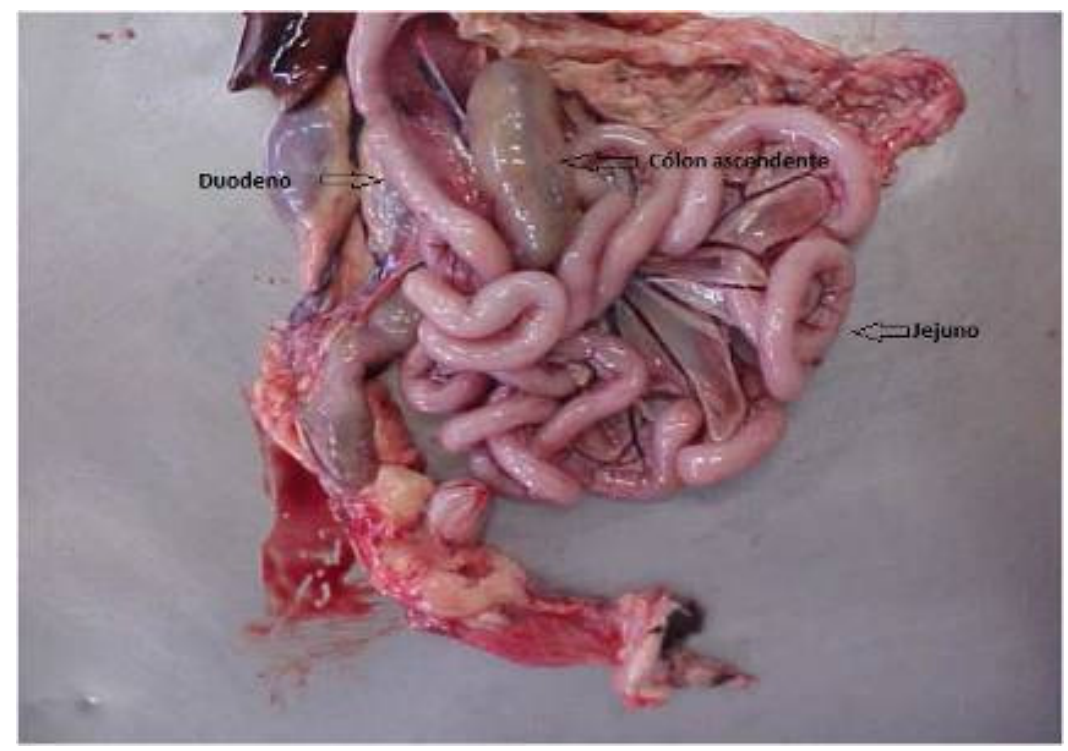

Figura 1- Intestino de gato doméstico (Felis catus domesticus)

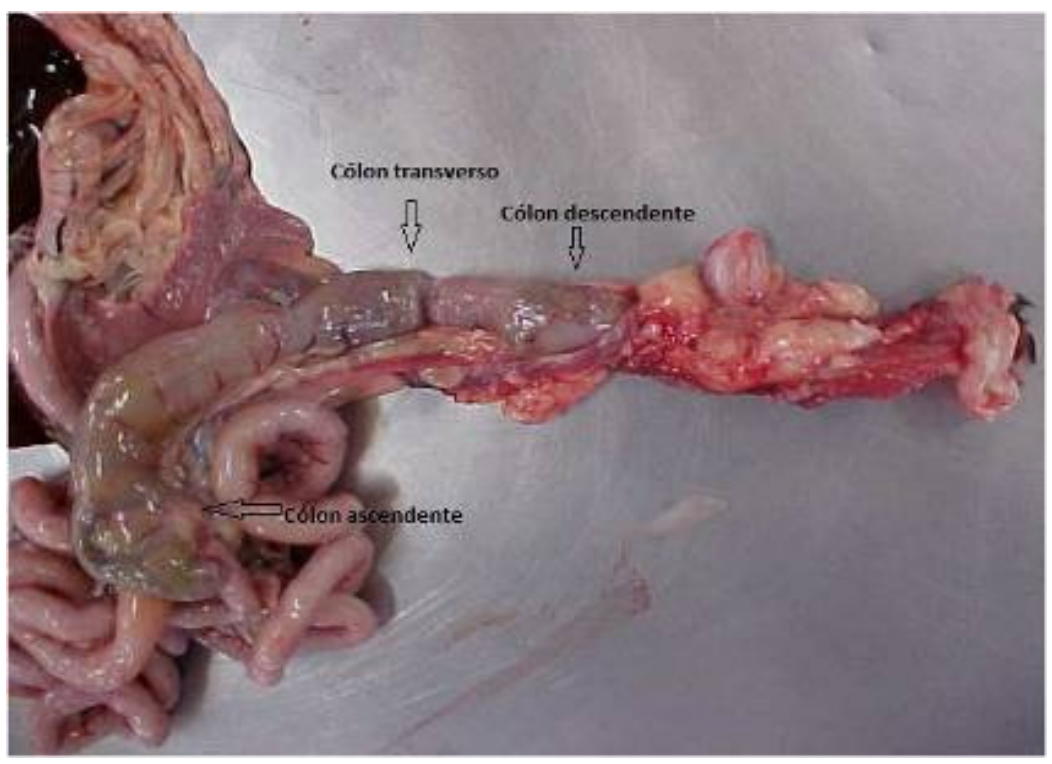

Figura 2 - Intestino grosso de gato doméstico (Felis catus domesticus). 
Tabela 2 - Comprimento médio em centímetros dos diversos segmentos intestinais e comprimento total médio do intestino de gatos machos e fêmeas $(n-8)$

\begin{tabular}{lcc}
\hline & Machos & Fêmeas \\
\hline Duodeno & 10,06 & 8,35 \\
Jejuno & 118,67 & 100,45 \\
Íleo & 2,69 & 2,25 \\
Ceco & 1,71 & 1,65 \\
Cólon ascendente & 5,09 & 3,9 \\
Cólon transverso & 2,07 & 1,57 \\
Cólon descendente & 10,27 & 8,29 \\
Reto & 3,86 & 3,11 \\
\hline Comprimento total & $\mathbf{1 6 4 , 9}$ & $\mathbf{1 2 9 , 5 7}$ \\
\hline
\end{tabular}

\section{CONCLUSÕES}

O gato doméstico apresenta o jejuno como segmento mais longo do trato digestivo, seguido pelo cólon descendente e pelo duodeno. Os machos apresentaram um maior comprimento intestinal que as fêmeas.

\section{REFERÊNCIAS}

CROMPTON, D. W.; NESHEIM, M. C. Host parasite relationships in the alimentary tract of domestics birds. Advances in Parasitology, v. 14, p. 95-194, 1976.

DYCE, K.M; SACK, W.O; WENSING, C.J.G. Tratado de Anatomia Veterinária. Rio de Janeiro, Guanabara Koogan, 1990, 567p.

GETTY, R. Anatomia dos animais domésticos. 5 ed. Rio de Janeiro Guanabara Koogan, v.2, 1981, 2000p.

GONZÁLEZ-JIMÉNEZ, E. Digestive physiology and feeding of capibaras. In: Rechcige, M. (Ed.). Handbook series in nutrition and food. CRC Press, Cleveland, USA, p. 167-177, 1977.

METTRICK, D. F.; PODESTA, R. B. Ecological and physiological aspects of helminth-host interactions in the mammalian gastrointestinal canal. Advances in Parasitology, v.12, p. 183-278, 1974. 
SANTOS, A.L.Q. et al. Comprimentos parciais e totais de intestino de gatos domésticos Felis catus domesticus - (Linnaeus, 1758). PUBVET, Londrina, V. 5, N. 14, Ed. 161, Art. 1092, 2011.

PAIVA, O. M.; BORELLI, V.; PEDUTTI NETO, J. Comprimento total do intestino em bovinos de origem européia. Revista da Faculdade de Medicina Veterinária e Zootecnia, Universidade de São Paulo, v.14, n.2, p.189-197, 1977.

PEREIRA, J. G. L.; et al. Comprimento total do intestino de suínos sem raça definida. Revista da Faculdade de Medicina Veterinária e Zootecnia, Universidade de São Paulo, v.23, n.1, p.25-26, 1986.

PEREIRA, J. G. L.; et al. Comprimento total do intestino em ratão do banhado (Myocastor coypus). In: ANAIS DO CONGRESSO LUSO BRASILEIRO DE ANATOMIA e IV CONGRESSO BRASILEIRO DE ANATOMIA. São Paulo, Brasil. 1988, 93p.

REECE, W. O. Fisiologia de animais domésticos. Roca LTDA, São Paulo, Brasil, 1996, 351p.

SANTOS, A.L.Q.; et al. Total and partial lengths of Oryctolagus cuniculus intestine. In: XVI PANVET, Santa Cruz de la Sierra. Anais..., 1998, 212p. 